\title{
Numerical computation for the chemotaxis model
}

\author{
Charazed Messikh ${ }^{1}$ and Amar Guesmia ${ }^{2}$ \\ ${ }^{1}$ Department of Mathematics, Badji Mokhtar University, \\ Faculty of sciences, 23000 Annaba, Algeria \\ ${ }^{2}$ Faculty of sciences, Department of mathematics, \\ University of 20 August 1955, 21000 Skikda, Algeria
}

abstract- In this paper, we present an implicit finite volume method for the space fractional Chemotaxis model on a finite domain. Our method is a numerical method which is based on the fractionally-shifted Grünwald formulas. This formula is used to discretisize the fractional derivative. We report several numerical experiments illustrating the efficiency of our method.

Keywords - Chemotaxis model, fractional derivative, finite volume, fractionally-shifted Grünwald.

\section{INTRODUCTION}

Chemotaxis is an important means for cellular communication. It is the influence of chemical substances in the environment on the movement of mobile species. This can lead to strictly oriented movement or to partially oriented and partially tumbling movement. Positive chemotaxis is the movement towards a higher concentration of the chemical substance whereas the movement towards regions of lower chemical concentration is called negative chemotactical movement.

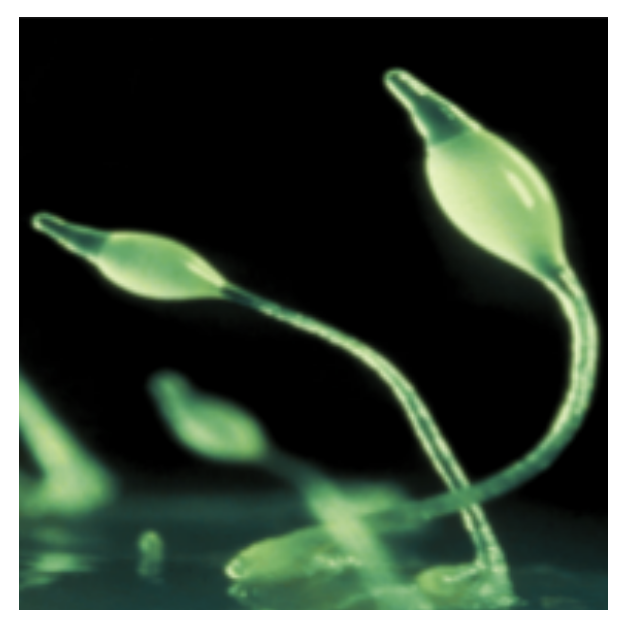

Figure 1: dictyostelum cell

An example is given by the model laboratory organism "Dictyostelium discoideum" which is found on dead leaves in forests and feeds on bacteria and yeasts. In the case of nutritional deficiency, this amoeba secretes a chemo-attractant to form a pseudo-plasmode (Figure 1) resembling a small slug and consisting of thousands of agglomerated amoebas. This pseudo- plasmode may persist for several days in order to seek more favorable nutritional conditions [3].

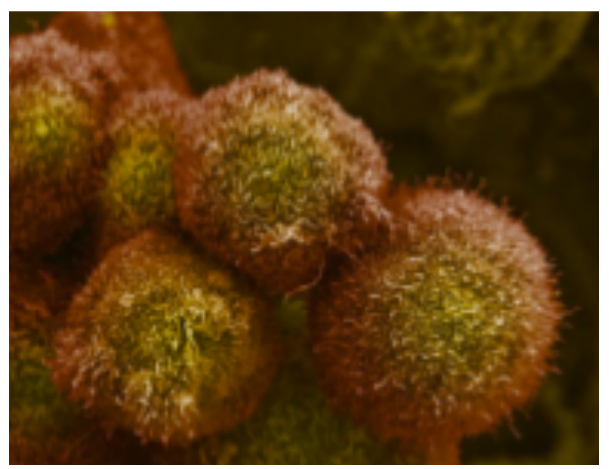

Figure 2: cancerous cells

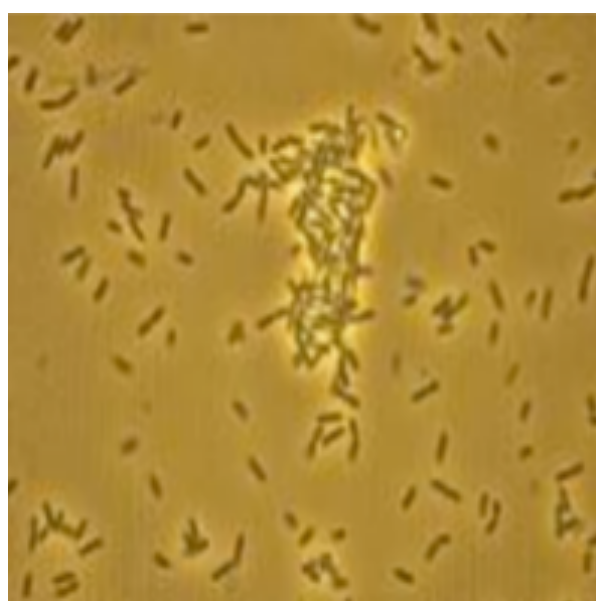

Figure 3: Bacillus bacteria

The second example is the cancerous cells that appear as a result of the mutation of healthy cells in the 
body that begin to multiply in a fast and uncontrolled way. Chemotherapy has the ability to slow the development of cancer cells and prevent angiogenesis (Figure 2) [3].

Another example is provided by the bacteria (Bacillus subtilis) which insure their nutrients by moving towards them. dioxygen-rich media (Figure 3), which necessitates a deep understanding of biological movements directed towards certain chemical species present in the environment as well as the development of appropriate tools for numerical simulations [3].

The classical chemotaxis model defined in Ref. 1 was first introduced by Paltak [12] in 1953, E. Keller and L. Segel [7] in 1970. It is given by a set of partial differential equations

$$
\begin{array}{ll}
u_{t}-\nabla(m \nabla u)+\nabla(\xi u \nabla c)=0, & (x, t) \in \mathbb{R}^{d} \times \mathbb{R}^{+}, \\
\delta c_{t}-\Delta c+\tau c+\rho u=0, & (x, t) \in \mathbb{R}^{d} \times \mathbb{R}^{+},
\end{array}
$$

where $u(x, t)$ denotes the density of bacteria in the position $x \in \mathbb{R}^{d}$ at time $t, c$ is the concentration of chemical signal substance, $\delta \geq 0$ represents the relaxation time, the parameter $\xi$ is the sensitivity of cells to the chemoattractant and $m, \tau$ and $\rho$ are given smooth functions.

Chemotaxis within complex and non-homogeneous media is not adequately described by the classical theory of Brownian motion and Fick's law. So, it can not be represented by the classical diffusion equation. It is a known mathematical fact that the Riesz operator, with $1<\alpha<2$ is less regularizing than the Laplacian. Thus, it is better modeled via fractional operators. This would imply a new breakdown of the Chemotaxis law for an important number of situations.

The field of fractional order derivatives has attracted the attention of many researchers in all branches of sciences and engineering. In recent years, many fields in sciences and technology have used fractional order derivatives to model many real world problems in their respective fields, as it has been revealed that these fractional order derivatives are very efficient in describing such problems (see [1, 2, 13]).

The numerical studies for solving fractional differential equations were discussed by several authors and by different methods [4,-6, 9, 10]. These methods are based on standard and Grünwald shifts formula which plays an important role in discretisation of the differential terms.

In this paper, we focus on the space fractional chemotaxis (SFC) model with a source term, which is obtained from the classical diffusion equation of this system by replacing the space derivatives with a generalized derivative of fractional order:

$$
\begin{array}{r}
\left.u_{t}-D^{\alpha} u+\frac{\partial}{\partial x}\left(u \frac{\partial c}{\partial x}\right)=S(x, t, \alpha), \quad(x, t) \in\right] a b[\times(0 T], \\
\left.-\frac{\partial^{2} c}{\partial x^{2}}+\tau c=0, \quad x \in\right] a b[
\end{array}
$$

with boundary and initial conditions

$$
\begin{array}{r}
u(a, t)=u(b, t)=0, \quad \text { for all } t \in[0 T], \\
c(a)=\gamma, c(b)=\beta, \\
u(x, 0)=u_{0}(x), \quad \text { for all } x \in[a b],
\end{array}
$$

where $u(x, t)$ is the cell density in the position $x \in[a b]$ at time $t, c$ the chemical density, the function $S$ regulates the cell die/divide, which controls the gross cell number in our observation and the positive constant $\tau$ the rate of attractant depletion, the positives constants $\gamma, \beta$ and $1<\alpha<2$ are given and $u_{0}$ is a smooth given function. The terms in equation (2) include the diffusion of the cells and chemotactic drift. On the other side, the equation (3) expresses the production of attractant [8]. The symbol $D^{\alpha} u$ stands for left Riemann-Liouville fractional derivative.

Definition I.1 (Riemann-Liouville fractional derivative on $[a, b])$

$$
\left.D^{\alpha} u(x, t)=\frac{\partial^{\alpha} u(x, t)}{\partial x^{\alpha}}=\frac{\partial^{2}}{\partial x^{2}}\left(I^{2-\alpha}\right) u(x, t) \text { for } \alpha \in\right] 12[,
$$

where $I^{\alpha}($.$) is called the Liouville integral and it is given$ by the following integral

$$
I^{\alpha} u(x, t)=\frac{1}{\Gamma(\alpha)} \int_{a}^{x} \frac{u(\xi, t)}{(x-\xi)^{1-\alpha}} d \xi,
$$

where $\Gamma($.$) is the well known Gamma function.$

In this paper, we derive an implicit finite volume method for solving (2 6) that utilizes a fractionallyshifted Grünwald formula for the discretisation of the fractional derivative. The remaining of the paper is organized as follows. In section II we investigate the implicit finite volume method for (2 6). In section III we give stability and convergence results . Finally, in section IV, numerical simulations illustrate our error bounds.

\section{Discretization METHOD OF THE PROBLEM}

In this section, we only discretize the equation (2) because the equation (3) with the boundary conditions $c(a)=\gamma$ and $c(b)=\beta$ cab be easy obtained in the one-dimensional case and its solution is given by

$$
c(x)=A \mathrm{e}^{\sqrt{\tau} x}+B \mathrm{e}^{-\sqrt{\tau} x} \in C^{\infty}([a b]),
$$


where

$$
\begin{aligned}
& A=\left(\gamma \mathrm{e}^{-\sqrt{\tau} b}-\beta \mathrm{e}^{-\sqrt{\tau} a}\right) /\left(\mathrm{e}^{\sqrt{\tau}(a-b)}-\mathrm{e}^{-\sqrt{\tau}(a-b)}\right) \\
& B=\left(\beta \mathrm{e}^{\sqrt{\tau} a}-\gamma \mathrm{e}^{\sqrt{\tau} b}\right) /\left(\mathrm{e}^{\sqrt{\tau}(a-b)}-\mathrm{e}^{-\sqrt{\tau}(a-b)}\right) .
\end{aligned}
$$

We can rewrite equation (2) as follows:

$$
u_{t}(x, t)=-\frac{\partial \Phi}{\partial x}+S(x, t, \alpha),
$$

where the total flux is given by

$$
\Phi(x, t)=q(x, t)+u(x, t) \frac{\partial c(x)}{\partial x},
$$

with the diffusive component

$$
\begin{aligned}
q(x, t) & =-\frac{\partial^{\alpha-1} u(x, t)}{\partial x^{\alpha-1}} \\
& \left.=-\frac{\partial}{\partial x}\left(I^{2-\alpha}\right) u(x, t), \alpha \in\right] 12[
\end{aligned}
$$

and advective component

$$
u(x, t) \frac{\partial c(x)}{\partial x}
$$

We consider a domain $\left[\begin{array}{ll}a & b\end{array}\right]$ that is discretized in $N+1$ uniformly spaced nodes $x_{i}=a+i h$ for $i=0, \ldots, N$, with the spatial step $h=(b-a) / N$. A finite volume discretization is applied by integrating over the $i$ th control volume $K_{i}=\left[x_{i-1 / 2}, x_{i+1 / 2}\right]$, that is,

$$
\begin{aligned}
\frac{d}{d t} \int_{x_{i-\frac{1}{2}}}^{x_{i+\frac{1}{2}}} u(x, t) d x= & \Phi\left(x_{i-\frac{1}{2}}, t\right)-\Phi\left(x_{i+\frac{1}{2}}, t\right) \\
& +\int_{x_{i-\frac{1}{2}}}^{x_{i+\frac{1}{2}}} S(x, t, \alpha) d x .
\end{aligned}
$$

Denoting the control volume averages $u$ by $\bar{u}=$ $\frac{1}{h} \int_{x_{i-1 / 2}}^{x_{i-1 / 2}} u d x \quad\left(\bar{S}=\frac{1}{h} \int_{x_{i-1 / 2}}^{x_{i+1 / 2}} S d x\right)$, and noting that no approximation has been introduced up to this point. So, we can write

$\frac{d}{d t} \bar{u}\left(x_{i}, t\right)=\frac{1}{h}\left[\Phi\left(x_{i-1 / 2}, t\right)-\Phi\left(x_{i+1 / 2}, t\right)+h \bar{S}\left(x_{i}, t, \alpha\right) d x\right]$.

The proposed method to approximate the diffusive flux $q\left(x_{i \mp 1 / 2}, t\right)$ is the fractionally-shifted Grünwald formulas which is given by the following definition:

Definition II.1 Shifted Grünwald formula on $\left[\begin{array}{ll}a & b\end{array}\right]$ )

$$
\frac{\partial^{\alpha} f(x, t)}{\partial x^{\alpha}} \approx \frac{1}{h^{\alpha}} \sum_{j=0}^{[(x-a) / h+p]} w_{j}^{\alpha} f(x-(j-p) h, t),
$$

where $p$ is the shift value and $w_{j}^{\alpha}$ are weight functions such that

$$
w_{0}^{\alpha}=1, \quad w_{j}^{\alpha}=(-1)^{j} \frac{\alpha(\alpha-1) \cdots(\alpha-j+1)}{j !}, j=1,2, \cdots
$$

For $p=0$ the equation $(18)$ is called the standard Grünwald formula. In the present method, the fractional shift $p=1 / 2$ is used in (18), allowing us to build approximations of the fractional derivatives at control volume faces $x_{i \mp 1 / 2}$ in terms of function values at the nodes $x_{i}$. This leads to the following diffusive flux approximation:

$$
\frac{\partial^{\alpha-1} u\left(x_{i-\frac{1}{2}}, t\right)}{\partial x^{\alpha-1}} \approx \frac{1}{h^{\alpha-1}} \sum_{j=0}^{i} w_{j}^{\alpha-1} u\left(x_{i-j}, t\right),
$$

at face $x_{i-\frac{1}{2}}$ and

$$
\frac{\partial^{\alpha-1} u\left(x_{i+\frac{1}{2}}, t\right)}{\partial x^{\alpha-1}} \approx \frac{1}{h^{\alpha-1}} \sum_{j=0}^{i+1} w_{j}^{\alpha-1} u\left(x_{i-j+1}, t\right),
$$

at face $x_{i+\frac{1}{2}}$.

Meanwhile, using a standard averaging scheme to discretize the advective flux (15), we can write

$$
u\left(x_{i \mp 1 / 2}, t\right) \frac{\partial c\left(x_{i \mp 1 / 2, t}\right)}{\partial x} \approx \frac{c_{i \mp 1 / 2}^{\prime}}{2}\left(u\left(x_{i \mp 1}, t\right)+u\left(x_{i}, t\right)\right),
$$

at face $x_{i \mp 1 / 2}$ and $c_{i \mp \frac{1}{2}}^{\prime}$ denotes the derivative of $c$ at face $x_{i \mp \frac{1}{2}}$ of the control volume $K_{i}$.

Using the standard control volume approximations, the control volume averaged terms can be approximated by nodal terms as follows:

$$
\overline{u_{i}} \approx u\left(x_{i}, t\right), \text { and } \overline{S_{i}} \approx S\left(x_{i}, t, \alpha\right)
$$

which completes the spatial discretisation. Let define a temporal partition $t_{n}=n k$ for $n=0,1, \ldots$, where $k$ is the time step, and approximate the temporal derivative in (17) by the standard first order backward difference. Let $u_{i}^{n} \approx u\left(x_{i}, t_{n}\right)$ as the numerical solution and $S_{i}^{n} \approx S\left(x_{i}, t_{n}\right)$, (noting $u_{0}=0$ ). combining the relations 2022 yields the total implicit scheme

$$
\begin{gathered}
\frac{u_{i}^{n+1}-u_{i}^{n}}{k}=\frac{1}{h}\left[-\frac{\dot{c}_{i+1 / 2}}{2} u_{i+1}^{n+1}+\left(\frac{\dot{c}_{i-1 / 2}-\dot{c}_{i+1 / 2}}{2}\right) u_{i}^{n+1}+\frac{\dot{c}_{i-1 / 2}}{2} u_{i-1}^{n+1}\right] \\
+\frac{1}{h}\left[\frac{1}{h^{\alpha-1}} \sum_{j=0}^{i} w_{j}^{\alpha-1}\left(u_{i-j+1}^{n+1}-u_{i-j}^{n+1}\right)+h S_{i}^{n+1}\right] \\
=\frac{1}{h} \sum_{j=0}^{N} g_{i j} u_{j}^{n+1}+S_{i}^{n+1}
\end{gathered}
$$

such that

$$
g_{i j}=\left\{\begin{array}{lr}
h^{1-\alpha}\left(w_{i-j+1}^{\alpha-1}-w_{i-j}^{\alpha-1}\right) & j<i-1 \\
\frac{\hat{c}_{i-1 / 2}}{2}+h^{1-\alpha}\left(w_{2}^{\alpha-1}-w_{1}^{\alpha-1}\right) & j=i-1 \\
\frac{c_{i-1 / 2}-\hat{c}_{i+1 / 2}}{2}+h^{1-\alpha}\left(w_{1}^{\alpha-1}-w_{0}^{\alpha-1}\right) & j=i \\
-\frac{\hat{c}_{i+1 / 2}}{2}+h^{1-\alpha} w_{0}^{\alpha-1} & j=i+1 \\
0 & j>i+1 .
\end{array}\right.
$$


If we define the numerical solution vector $U^{n}=$ $\left(u_{1}^{n}, \ldots, u_{N-1}^{n}\right)$ then the equation 23 is written in matrix form as follows:

$$
\left(I+\frac{k}{h} A\right) U^{n+1}=U^{n}+k S^{n+1}
$$

such that the elements of the matrix $A$ are $-g_{i j}$ and the elements of the vector $S^{n+1}$ are $S_{i}^{n+1}$.

\section{Scheme Analysis}

The scheme 25) can be written in matrix form in a straightforward manner:

$$
U^{n+1}=M\left(U^{n}+k S^{n+1}\right),
$$

where

$$
M=\left(I+\frac{k}{h} A\right)^{-1}
$$

is the iteration matrix. The following Theorem gives us the condition on the constants $\gamma$ and $\beta$ under which the function derivative function of $c(x)$ does not change its sign.

Theorem III.1 Let $1<\alpha<2, \gamma>0, \beta>0$, $a$ and $b$ are constants. Then $c^{\prime}(x)$ is increasing function. Moreover i) If

$$
\frac{\beta}{\gamma}<\frac{2}{\exp (\sqrt{\tau}(b-a)+\exp (-\sqrt{\tau}(b-a)},
$$

then $c^{\prime}(x)$ is negative.

ii) If

$$
\frac{\beta}{\gamma}>\frac{\exp (\sqrt{\tau}(b-a))+\exp (-\sqrt{\tau}(b-a))}{2},
$$

then $c^{\prime}(x)$ is positive.

\section{Stability [11]}

The following Theorem gives us the conditions under the matrix's elements $g_{i j}$ in (24) for that this matrix become strictly diagonally dominant.

Theorem III.2 Let $\beta>0, \gamma>0,1<\alpha<2$ and $c^{\prime}$ satisfies

$$
\left\{\begin{array}{l}
\text { i) } \frac{c^{\prime}(a)}{2\left(w_{1}^{\alpha-1}-w_{2}^{\alpha-1}\right)} \leq h^{1-\alpha} \text { if } \frac{\beta}{\gamma}<\frac{2}{\exp (\sqrt{\tau}(b-a)+\exp (-\sqrt{\tau}(b-a)}, \\
\text { ii) } \frac{c^{\prime}(b)}{2} \leq h^{1-\alpha} \text { if } \frac{\beta}{\gamma}>\frac{\exp (\sqrt{\tau}(b-a))+\exp (-\sqrt{\tau}(b-a))}{2}
\end{array}\right.
$$

where $w_{1}^{\alpha-1}$ and $w_{2}^{\alpha-1}$ are defined in equation [19]. Then the coefficients $g_{i j}$ in (24) satisfy

$$
\left|g_{i i}\right|>\sum_{j=0, j \neq i}^{j=N}\left|g_{i j}\right|, \quad i=1, \ldots N .
$$

Remark III.1 We remark that diagonal elements of the matrix $A$ are positive and moreover from Theorem 3.4, it is strictly diagonally dominant. Then the iteration matrix $M$ in scheme (23) is convergent. Thus we deduce that the scheme itself is unconditionally stable (i.e. $\varrho(M)<1$ ).

\section{Convergence [11]}

In order to state our convergence result, we first need the following Theorem:

Theorem III.3 (see [6]) Let $\alpha$ and $p$ be positive numbers, and suppose that $f \in C^{[\alpha]+\eta+2}(\mathbb{R})$ and all derivatives of $f$ up to order $[\alpha]+\eta+2$ belong to $L^{1}(\mathbb{R})$. Define

$$
\Delta_{h, p}^{\alpha} f(x)=\sum_{j=0}^{\infty}(-1)^{j}\left(\begin{array}{c}
\alpha \\
j
\end{array}\right) f(x-(j-p) h) .
$$

Then if $a=-\infty$ and $b=\infty$ in (7), there exist constants $C_{\ell}$ independent of $h, f$ and $x$ such that

$$
h^{-\alpha} \Delta_{h, \alpha}^{\alpha} f(x)=\frac{\partial^{\alpha} f}{\partial x^{\alpha}}+\sum_{\ell=1}^{\eta-1} c_{\ell} \frac{\partial^{\alpha+\ell} f(x)}{\partial x^{\alpha+\ell}}+0\left(h^{\eta}\right)
$$

is uniformly in $x \in \mathbb{R}$.

Remark III.2 The relation in Theorem 3.6 remains again valid on the finite domain $\left[\begin{array}{ll}a & b\end{array}\right]$ for function that vanishes at extremity of the interval, due to the fact that we can put it equal to zero outside this interval.

Using Theorem 3.6 we may rewrite $(20)$ and 21 with the error terms:

$$
\begin{aligned}
\frac{\partial^{(\alpha-1)} u\left(x_{i-1 / 2}, t\right)}{\partial x^{(\alpha-1)}}= & \frac{1}{h^{\alpha-1}} \sum_{j=0}^{i} w_{j}^{\alpha-1} u\left(x_{i-j}, t\right) \\
& -C_{1} \frac{\partial^{\alpha} u\left(x_{i-1 / 2}, t\right)}{\partial x^{\alpha}} h+0\left(h^{2}\right), \\
\frac{\partial^{(\alpha-1)} u\left(x_{i+1 / 2}, t\right)}{\partial x^{(\alpha-1)}=} & \frac{1}{h^{\alpha-1}} \sum_{j=0}^{i+1} w_{j}^{\alpha-1} u\left(x_{i-j}, t\right) \\
& -C_{1} \frac{\partial^{\alpha} u\left(x_{i+1 / 2}, t\right)}{\partial x^{\alpha}} h+0\left(h^{2}\right) .
\end{aligned}
$$

For discretisation the advection term $u \frac{\partial c}{\partial x}$, we propose the averaging scheme for $u$ at the face $x_{i \pm \frac{1}{2}}$

$$
u\left(x_{i \pm 1 / 2}\right)=\frac{1}{2}\left(u\left(x_{i}, t\right)+u\left(x_{i \pm 1}, t\right)\right)+0\left(h^{2}\right),
$$

and the control volume averages are obtained as

$$
\begin{gathered}
\frac{\partial \bar{u}_{i}\left(t_{n+1}\right)}{\partial t}=\frac{u\left(x_{i}, t_{n+1}\right)-u\left(x_{i}, t_{n}\right)}{k}+0\left(\tau+h^{2}\right), \\
\bar{S}_{i}\left(t_{n+1}\right)=S\left(x_{i}, t_{n+1}\right)+0\left(h^{2}\right) .
\end{gathered}
$$

Theorem III.4 The numerical scheme 23] is convergent of order 1 in space and time. 


\section{Numerical Results}

in this section, we compare the numerical solutions with the exact solutions of the following SFC problem with $\tau=0.01$ and $\alpha=1.5$.

$$
\begin{array}{r}
\left.u_{t}-D^{\alpha} u+\frac{\partial}{\partial x}\left(u \frac{\partial c}{\partial x}\right)=S(x, t, \alpha),(x, t) \in\right] a b[\times] 01[, \\
\left.-\frac{\partial^{2} c}{\partial x^{2}}+\tau c=0, \quad x \in\right] a b[
\end{array}
$$

with $\tau=0.01$, boundary and initial conditions

$$
\begin{array}{r}
u(a, t)=u(b, t)=0, \quad \text { for all } t \in[01], \\
c(a)=\gamma, c(b)=\beta,
\end{array}
$$

The goal of these experiments is to show that the convergence and stability results derived in section (3) are realized. For this, we begin by giving two numerical experiments of the proposed problem; the first verified the condition (i) and the second verified the condition (ii) in Theorem (3.4). We compare the numerical solution of these experiments with their exact solutions . Let us $a=-1$ and $b=1$. The exact solution of the above system is given by

$$
\left\{\begin{array}{l}
u(t, x)=\left(1-x^{2}\right) e^{-t} \\
c(x)=A \mathrm{e}^{\sqrt{\tau} x}+B \mathrm{e}^{-\sqrt{\tau} x}
\end{array}\right.
$$

where

$$
\begin{gathered}
A=\left(\gamma \mathrm{e}^{-\sqrt{\tau} b}-\beta \mathrm{e}^{-\sqrt{\tau} a}\right) /\left(\mathrm{e}^{\sqrt{\tau}(a-b)}-\mathrm{e}^{-\sqrt{\tau}(a-b)}\right), \\
B=\left(\beta \mathrm{e}^{\sqrt{\tau} a}-\gamma \mathrm{e}^{\sqrt{\tau} b}\right) /\left(\mathrm{e}^{\sqrt{\tau}(a-b)}-\mathrm{e}^{-\sqrt{\tau}(a-b)}\right) .
\end{gathered}
$$

With the source

$$
S(t, x, \alpha)=e^{-t}\left[S_{1}(x, \alpha)-S_{2}(x, \alpha)\right]
$$

such that

$$
\begin{aligned}
S_{1}(x, \alpha)= & -2 x \sqrt{\tau}\left(A e^{\sqrt{\tau} x}-B e^{-\sqrt{\tau} x}\right) \\
& +\tau\left(1-x^{2}\right)\left(A e^{\sqrt{\tau} x}+B e^{-\sqrt{\tau} x}\right), \\
S_{2}(x, \alpha)= & \left(1-x^{2}\right)+\frac{2(x+1)^{(1-\alpha)}}{\Gamma(2-\alpha)}-2 \frac{(x+1)^{(2-\alpha)}}{\Gamma(3-\alpha)} .
\end{aligned}
$$

- Experiment 1 (condition (i) in Theorem(3.4)): We consider the problem (4.1-4.5) with $\beta=1$ and $\gamma=2$. We note that these constants verify the condition (i) in Theorem (3.4) for $\alpha=1.5$. We choose the spatial step $h$ such that $h<\left(\frac{2\left(w_{1}^{0.5}-w_{2}^{0.5}\right)}{c^{\prime}(a)}\right)^{2}$. In this experiment, we can take $h=0.1$ so $N=100$.
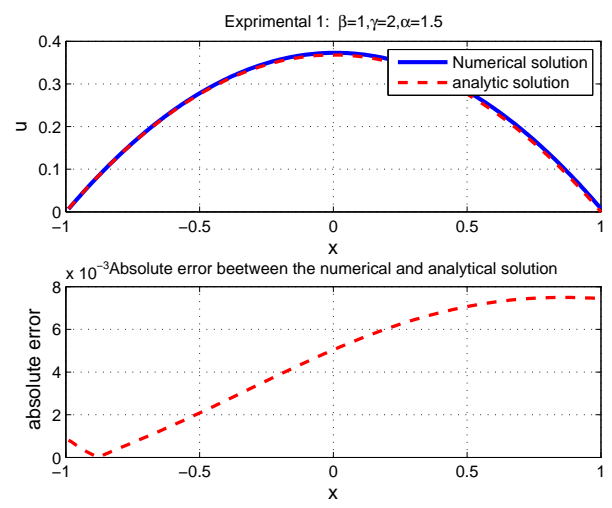

Figure 4: Comparison between numerical and exact solutions for $\gamma=2, \beta=1$ and $\alpha=1.5$.

- Experiment 2 (condition (ii) in Theorem(3.4)): We consider the problem (4.1-4.5) with $\beta=2$ and $\gamma=1$. We note that theses constants verify the condition (ii) in Theorem (3.4) for $\alpha=1.5$. We choose the spatial step $h$ such that $h<\left(\frac{2}{c^{\prime}(b)}\right)^{2}$. In this experiment, we can take $h=0.1$ so $N=100$.
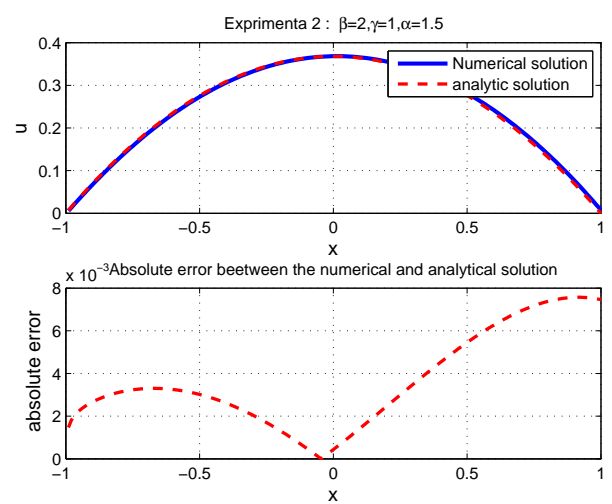

Figure 5: Comparison between numerical and exact solutions for $\gamma=1, \beta=2$ and $\alpha=1.5$.

\section{Discussion:}

- The figure 4 shows that the absolute error, the difference between the exact solution and the approximation solution obtained from finite volume method, at $x=-0.99$ equal to $8 \times 10^{-3}$. This absolute error vanishes at $x=-0.88$ and then increases up to $7.5 \times 10^{-3}$ and finally decrease to the value $7.4 \times 10^{-3}$ at the neighbor of $x=1$. Thus, the maximum absolute error is $7.5 \times 10^{-3}$. So, we conclude that the analytic and the approximated solutions are almost identical.

- Similarly, the figure 5 shows that the absolute error, the difference between the exact solution 
and the approximation solution obtained from finite volume method, at $x=-0.99$ equal to $1.5 \times 10^{-3}$. It increases up to $3.3 \times 10^{-3}$ and decreases until it vanishes at $x=-0.05$. Then, it starts to increases up to the value $7.6 \times 10^{-3}$ and finally, it decreases to the value $7.5 \times 10^{-3}$ at the neighbor of $x=1$. Thus, the maximum absolute error $7.6 \times 10^{-3}$. Thus, we conclude that the analytic and the approximated solutions are almost identical.

\section{REFERENCES}

[1] Nikolaos Bournaveas and Vincent Calvez, The onedimensional keller segel model with fractional diffusion of cells, Nonlinearity 23 (2010), no. 4, 923.

[2] Carlos Escudero, The fractional keller segel model, Nonlinearity 19 (2006), no. 12, 2909.

[3] G Chamoun Mathematical and numerical study of chemotaxis-fluid models and application in biology,Theses, https://tel.archives-ouvertes.fr/tel01015918/file/manuscrit-Chamoun.pdf, 2014.

[4] A Guesmia and N Daili, Numerical approximation of fractional burgers equation, Communications in Mathematics and Applications 1 (2010), no. 2, 77-90.

[5] Hala Hejazi, Tim Moroney, and Fawang Liu, A comparison of finite difference and finite volume methods for solving the space-fractional advection-dispersion equation with variable coefficients, ANZIAM Journal $\mathbf{5 4}$ (2013), 557-573.
[6] Hala Hejazi, Timothy J. Moroney, and Fawang Liu, Stability and convergence finite volume method for the space fractional advection dispersion equation of $a$, Journal of Computational and Applied Mathematics 255 (2014), 684-697.

[7] Evelyn F Keller and Lee A Segel, Initiation of slime mold aggregation viewed as an instability, J. Theor. Biol. 26 (1970), 399-415.

[8] A Marrocco, Numerical simulation of chemotactic bacteria aggregation via mixed finite elements, ESAIM: Mathematical Modelling and Numerical Analysis 37 (2003), no. 4, 617-630.

[9] Mark M Meerschaert and Charles Tadjeran, Finite difference approximations for fractional advection dispersion flow equations, Journal of Computational and Applied Mathematics 172 (2004), no. 1, 65-77.

[10] Mark M Meerschaert and Charles Tadjeran, Finite difference approximations for two-sided space-fractional partial differential equations, Applied Numerical Mathematics 56 (2006), no. 1, 80-90.

[11] C Messikh, Study of the Stability and convergence of an implicit finite volume method for an spatial fractional Keller-Segel model, ICMIT17 (IEEE Explorer) .

[12] Clifford S Patlak, Random walk with persistence and external bias, The bulletin of mathematical biophysics 15 (1953), no. 3, 311-338.

[13] I Podlubny, fractional derivative fractional, Academic Press New York, 1999. 